Pacific

Journal of

Mathematics

\title{
WEYL TRANSFORMS ASSOCIATED WITH A SINGULAR SECOND-ORDER DIFFERENTIAL OPERATOR
}

CYRINE BACCAR AND LAKHDAR TANNECH RACHDI 


\title{
WEYL TRANSFORMS ASSOCIATED WITH A SINGULAR SECOND-ORDER DIFFERENTIAL OPERATOR
}

\author{
CYRINE BACCAR AND LAKHDAR TANNECH RACHDI
}

\begin{abstract}
For a class of singular second-order differential operators $\Delta$, we define and study the Weyl transforms $W_{\sigma}$ associated with $\Delta$, where $\sigma$ is a symbol in $S^{m}$, for $m \in \mathbb{R}$. We give criteria in terms of $\sigma$ for boundedness and compactness of the transform $W_{\sigma}$.
\end{abstract}

\section{Introduction}

Herman Weyl [1931] studied extensively the properties of pseudodifferential operators arising in quantum mechanics, regarding them as bounded linear operators on $L^{2}\left(\mathbb{R}^{n}\right)$, the space of square-integrable functions on $\mathbb{R}^{n}$ with respect to Lebesgue measure). M. W. Wong calls these operators, which are the subject of his book [Wong 1998], Weyl transforms.

Here we consider the second-order differential operator defined on $] 0,+\infty[$ by

$$
\Delta u=u^{\prime \prime}+\frac{A^{\prime}}{A} u^{\prime}+\rho^{2} u,
$$

where $A$ is a nonnegative function satisfying certain conditions and $\rho$ is a nonnegative real number.

This operator plays an important role in analysis. For example, many special functions (orthogonal polynomials) are eigenfunctions of an operator of $\Delta$ type. The radial part of the Beltrami-Laplacian in a symmetric space is also of $\Delta$ type. Many aspects of such operators have been studied; we mention, in chronological order, [Chebli 1979; Trimèche 1981; Zeuner 1989; Xu 1994; Trimèche 1997; Nessibi et al. 1998]. In particular, the first two of these references investigate standard constructions of harmonic analysis, such as translation operators, convolution product, and Fourier transform, in connection with $\Delta$.

Building on these results, we define and study the Weyl transforms associated with $\Delta$, giving criteria for boundedness and compactness of these transforms. To obtain these results we first define the Fourier-Wigner transform associated with $\Delta$, and establish an inversion formula.

Keywords: Weyl transform, compact operator, Fourier-Wigner transform. 
More precisely, in Section 1 we recall some properties of harmonic analysis for the operator $\Delta$. In Section 2 we define the Fourier-Wigner transform associated with $\Delta$, study some of its properties, and prove an inversion formula.

In Section 3 we introduce the Weyl transform $W_{\sigma}$ associated with $\Delta$, with $\sigma$ a symbol in class $S^{m}$, for $m \in \mathbb{R}$, and we give its connection with the FourierWigner transform. We prove that, for $\sigma$ sufficiently smooth, $W_{\sigma}$ is a compact operator from $L^{2}(d v)$ (the space of square-integrable functions with respect to the measure $d \nu(x)=A(x) d x)$ into itself.

In Section 4 we define $W_{\sigma}$ for $\sigma$ in a certain space $L^{p}(d \nu \otimes d \gamma)$, with $p \in[1,2]$, and we establish that $W_{\sigma}$ is again a compact operator.

In Section 5 we define $W_{\sigma}$ for $\sigma$ in another function space, and use this to prove in Section 6 that for $p>2$ there exists a function $\sigma$ in the $L^{p}$ space corresponding to that of Section 4, with the property that the Weyl transform $W_{\sigma}$ is not bounded on $L^{2}(d v)$.

\section{The operator $\Delta$}

We consider the second-order differential operator $\Delta$ defined on $] 0,+\infty[$ by

$$
\Delta u=u^{\prime \prime}+\frac{A^{\prime}}{A} u^{\prime}+\rho^{2} u,
$$

where $\rho$ is a nonnegative real number and

$$
A(x)=x^{2 \alpha+1} B(x), \quad \alpha>-\frac{1}{2},
$$

for $B$ a positive, even, infinitely differentiable function on $\mathbb{R}$ such that $B(0)=1$. Moreover we assume that $A$ and $B$ satisfy the following conditions:

(i) $A$ is increasing and $\lim _{x \rightarrow+\infty} A(x)=+\infty$.

(ii) $\frac{A^{\prime}}{A}$ is decreasing and $\lim _{x \rightarrow+\infty} \frac{A^{\prime}(x)}{A(x)}=2 \rho$.

(iii) There exists a constant $\delta>0$ such that

$$
\begin{aligned}
& \frac{B^{\prime}(x)}{B(x)}=D(x) \exp (-\delta x) \quad \text { if } \rho=0, \\
& \frac{A^{\prime}(x)}{A(x)}=2 \rho+D(x) \exp (-\delta x) \quad \text { if } \rho>0,
\end{aligned}
$$

where $D$ is an infinitely differentiable function on $] 0,+\infty[$, bounded and with bounded derivatives on all intervals $\left[x_{0},+\infty\left[\right.\right.$, for $x_{0}>0$.

This operator was studied in [Chebli 1979; Nessibi et al. 1998; Trimèche 1981], and the following results have been established: 
(I) For all $\lambda \in \mathbb{C}$, the equation

$$
\left\{\begin{array}{l}
\Delta u=-\lambda^{2} u \\
u(0)=1, u^{\prime}(0)=0
\end{array}\right.
$$

admits a unique solution, denoted by $\varphi_{\lambda}$, with the following properties:

- $\varphi_{\lambda}$ satisfies the product formula

$$
\varphi_{\lambda}(x) \varphi_{\lambda}(y)=\int_{0}^{\infty} \varphi_{\lambda}(z) w(x, y, z) A(z) d z \quad \text { for } x, y \geq 0
$$

where $w(x, y, \cdot)$ is a measurable positive function on $[0,+\infty[$, with support in $[|x-y|, x+y]$, satisfying

$$
\begin{gathered}
\int_{0}^{\infty} w(x, y, z) A(z) d z=1, \\
w(x, y, z)=w(y, x, z) \quad \text { for } z \geq 0, \\
w(x, y, z)=w(x, z, y) \quad \text { for } z>0 ;
\end{gathered}
$$

- for $x \geq 0$, the function $\lambda \mapsto \varphi_{\lambda}(x)$ is analytic on $\mathbb{C}$;

- for $\lambda \in \mathbb{C}$, the function $x \mapsto \varphi_{\lambda}(x)$ is even and infinitely differentiable on $\mathbb{R}$;

- $\left|\varphi_{\lambda}(x)\right| \leq 1$ for all $\lambda \in \mathbb{R}$ and $x \in \mathbb{R}$;

- for $x>0$, and $\lambda>0$ we have

$$
\varphi_{\lambda}(x)=\frac{1}{\sqrt{B(x)}} j_{\alpha}(\lambda x)+A^{-1 / 2}(x) \theta_{\lambda}(x),
$$

where $j_{\alpha}$ is defined by $j_{\alpha}(0)=1$ and $j_{\alpha}(s)=2^{\alpha} \Gamma(\alpha+1) s^{-\alpha} J_{\alpha}(s)$ if $s \neq 0$ (with $J_{\alpha}$ the Bessel function of first kind), and the function $\theta_{\lambda}$ satisfies

$$
\left|\theta_{\lambda}(x)\right| \leq \frac{c_{1}}{\lambda^{\alpha+\frac{3}{2}}}\left(\int_{0}^{x}|Q(s)| d s\right) \exp \left(\frac{c_{2}}{\lambda} \int_{0}^{x}|Q(s)| d s\right)
$$

with $c_{1}, c_{2}$ positive constants and $Q$ the function defined on $] 0,+\infty[$ by

$$
Q(x)=\frac{\frac{1}{4}-\alpha^{2}}{x^{2}}+\frac{1}{4}\left(\frac{A^{\prime}(x)}{A(x)}\right)^{2}+\frac{1}{2}\left(\frac{A^{\prime}(x)}{A(x)}\right)^{\prime}-\rho^{2} .
$$

(II) For nonzero $\lambda \in \mathbb{C}$, the equation $\Delta u=-\lambda^{2} u$ has a solution $\Phi_{\lambda}$ satisfying

$$
\Phi_{\lambda}(x)=A^{-1 / 2}(x) \exp (i \lambda x) V(x, \lambda),
$$

with $\lim _{x \rightarrow+\infty} V(x, \lambda)=1$. Consequently there exists a function (spectral function)

$$
\lambda \mapsto c(\lambda),
$$


such that

$$
\varphi_{\lambda}=c(\lambda) \Phi_{\lambda}+c(-\lambda) \Phi_{-\lambda} \quad \text { for nonzero } \lambda \in \mathbb{C} .
$$

Moreover there exist positive constants $k_{1}, k_{2}, k_{3}$ such that

$$
k_{1}|\lambda|^{\alpha+1 / 2} \leq|c(\lambda)|^{-1} \leq k_{2}|\lambda|^{\alpha+1 / 2}
$$

for all $\lambda$ such that $\operatorname{Im} \lambda \leq 0$ and $|\lambda| \geq k_{3}$.

Notation. We denote by

- $d v(x)$ the measure defined on $[0,+\infty[$ by

$$
d v(x)=A(x) d x
$$

- $L^{p}(d v)$, for $1 \leq p \leq+\infty$, the space of measurable functions on $[0,+\infty[$ satisfying

$$
\begin{aligned}
\|f\|_{p, v}:= & \left(\int_{0}^{+\infty}|f(x)|^{p} d \nu(x)\right)^{1 / p}<+\infty \quad \text { for } 1 \leq p<+\infty, \\
& \|f\|_{\infty, v}:=\operatorname{ess~sup~}_{x \in[0,+\infty[}|f(x)|<+\infty
\end{aligned}
$$

- $d \gamma(\lambda)$ the measure defined on $[0,+\infty[$ by

$$
d \gamma(\lambda)=\frac{d \lambda}{2 \pi|c(\lambda)|^{2}}
$$

- $L^{p}(d \gamma)$, for $1 \leq p \leq+\infty$, the space of measurable functions on $[0,+\infty[$ satisfying $\|f\|_{p, \gamma}<+\infty$;

- $D_{*}(\mathbb{R})$ the space of even, infinitely differentiable functions on $\mathbb{R}$, with compact support;

- $\mathbb{H}_{*}(\mathbb{C})$ the space of even analytic functions on $\mathbb{C}$, rapidly decreasing of exponential type.

Definition 1.1. The translation operator associated with $\Delta$ is defined on $L^{1}(d v)$ by

$$
\mathscr{T}_{x} f(y)=\int_{0}^{+\infty} f(z) w(x, y, z) d v(z) \quad \text { for } x, y \geq 0,
$$

where $w$ is defined in (1-3). The convolution product associated with $\Delta$ is defined by

$$
(f * g)(x)=\int_{0}^{+\infty} \mathscr{T}_{x} f(y) g(y) d \nu(y) \quad \text { for } f, g \in L^{1}(d v) .
$$




\section{Properties of translation and convolution.}

- The translation operator satisfies

$$
\mathscr{T}_{x} \varphi_{\lambda}(y)=\varphi_{\lambda}(x) \varphi_{\lambda}(y) .
$$

- Let $f \in L^{1}(d v)$. Then

$$
\int_{0}^{+\infty} \mathscr{T}_{x} f(y) d v(y)=\int_{0}^{+\infty} f(y) d v(y) \quad \text { for } x \in[0,+\infty[
$$

and

$$
\left\|\mathscr{T}_{x} f\right\|_{1, v} \leq\|f\|_{1, v}
$$

- Let $f \in L^{p}(d v)$ with $1 \leq p \leq+\infty$. For all $x \in\left[0,+\infty\right.$ [, the function $\mathscr{T}_{x} f$ belongs to $L^{p}(d \nu)$ and

$$
\left\|\mathscr{T}_{x} f\right\|_{p, v} \leq\|f\|_{p, v} .
$$

- For $f, g \in L^{1}(d v)$ the function $f * g$ also lies in $L^{1}(d v)$. The convolution product is commutative and associative.

- For $f \in L^{1}(d v)$ and $g \in L^{p}(d v)$, with $1 \leq p<+\infty$, the function $f * g$ lies in $L^{p}(d v)$ and we have

$$
\|f * g\|_{p, v} \leq\|f\|_{1, v}\|g\|_{p, v} .
$$

- For $f, g$ even and continuous on $\mathbb{R}$, with supports

$$
\text { supp } f \subset[-a, a] \text { and } \operatorname{supp} g \subset[-b, b],
$$

the function $f * g$ is continuous on $\mathbb{R}$ and

$$
\operatorname{supp}(f * g) \subset[-a-b, a+b] .
$$

Definition 1.2. The Fourier transform associated with the operator $\Delta$ is defined on $L^{1}(d v)$ by

$$
\mathscr{F} f(\lambda)=\int_{0}^{+\infty} f(x) \varphi_{\lambda}(x) d \nu(x) \quad \text { for } \lambda \in \mathbb{R} .
$$

\section{Properties of the Fourier transform.}

- For $f \in L^{1}(d v)$ such that $\mathscr{F} f \in L^{1}(d \gamma)$, we have the inversion formula

$$
f(x)=\int_{0}^{+\infty} \mathscr{F} f(\lambda) \varphi_{\lambda}(x) d \gamma(\lambda) \quad \text { for a.e. } x \in[0,+\infty[.
$$

- For $f \in L^{1}(d v)$,

$$
\mathscr{F}\left(\mathscr{T}_{x} f\right)(\lambda)=\varphi_{\lambda}(x) \mathscr{F} f(\lambda) \text { for all } x \in[0,+\infty[\text { and } \lambda \in \mathbb{R} .
$$


- For $f, g \in L^{1}(d v)$,

$$
\mathscr{F}(f * g)(\lambda)=\mathscr{F} f(\lambda) \mathscr{F} g(\lambda) \text {. for all } \lambda \in[0,+\infty[.
$$

- F can be extended to an isometric isomorphism from $L^{2}(d \nu)$ onto $L^{2}(d \gamma)$. This means that

$$
\begin{aligned}
& \|\mathscr{F} f\|_{2, \gamma}=\|f\|_{2, v} \quad \text { for } f \in L^{2}(d v), \\
& \left\|\mathscr{F}^{-1} f\right\|_{2, v}=\|f\|_{2, \gamma} \quad \text { for } f \in L^{2}(d \gamma) \text {. }
\end{aligned}
$$

Proposition 1.3. Let $f$ be in $L^{p}(d v)$, with $p \in[1,2]$. Then $\mathscr{F} f$ belongs to $L^{p^{\prime}}(d \gamma)$, with $1 / p+1 / p^{\prime}=1$, and

$$
\|\mathscr{F} f\|_{p^{\prime}, \gamma} \leq\|f\|_{p, \nu} .
$$

Proof. Since $\left|\varphi_{\lambda}(x)\right| \leq 1$ for $\lambda \in \mathbb{R}$ and $x \in \mathbb{R}$, we get $\|\mathscr{F} f\|_{\infty, \gamma} \leq\|f\|_{1, \nu}$. This, together with (1-9) and the Riesz-Thorin Theorem [Stein 1956; Stein and Weiss 1971], shows that for under the conditions of the proposition $\mathscr{F} f$ belongs to $L^{p^{\prime}}(d \gamma)$ and satisfies (1-11).

From [Chebli 1979], the Fourier transform $\mathscr{F}$ is a topological isomorphism from $D_{*}(\mathbb{R})$ onto $\mathbb{H}_{*}(\mathbb{C})$ (see page 204 for notation). The inverse mapping is given by

$$
\mathscr{F}^{-1} f(x)=\int_{0}^{+\infty} f(\lambda) \varphi_{\lambda}(x) d \gamma(\lambda) \quad \text { for } x \in \mathbb{R} .
$$

\section{Fourier-Wigner transform associated with $\Delta$}

Definition 2.1. The Fourier-Wigner transform associated with the operator $\Delta$ is the mapping $V$ defined on $D_{*}(\mathbb{R}) \times D_{*}(\mathbb{R})$ by

$$
V(f, g)(x, \lambda)=\int_{0}^{+\infty} f(y) \mathscr{T}_{x} g(y) \varphi_{\lambda}(y) d \nu(y) \quad \text { for }(x, \lambda) \in \mathbb{R} \times \mathbb{R} .
$$

Remark. The transform $V$ can also be written in the forms

$$
V(f, g)(x, \lambda)=\mathscr{F}_{(}\left(f \mathscr{T}_{x} g\right)(\lambda)=\varphi_{\lambda} f * g(x) .
$$

Notation. We denote by

- $D_{*}\left(\mathbb{R}^{2}\right)$ the space of infinitely differentiable functions on $\mathbb{R}^{2}$, even with respect to each variable, with compact support;

- $S_{*}\left(\mathbb{R}^{2}\right)$ the space of infinitely differentiable functions on $\mathbb{R}^{2}$, even with respect to each variable, rapidly decreasing together with all their derivatives; 
- $L^{p}(d v \otimes d v)$, for $1 \leq p \leq+\infty$, the space of measurable functions on the product $[0,+\infty[\times[0,+\infty[$ satisfying

$$
\begin{aligned}
\|f\|_{p, \nu \otimes v} & :=\left(\int_{0}^{+\infty} \int_{0}^{+\infty}|f(x, y)|^{p} d v(x) d v(y)\right)^{1 / p}<+\infty \quad \text { for } 1 \leq p<+\infty, \\
\|f\|_{\infty, \nu \otimes v} & :=\operatorname{ess}_{x, y \in[0,+\infty[} \sup _{1}|f(x, y)|<+\infty
\end{aligned}
$$

- $L^{p}(d v \otimes d \gamma)$, for $1 \leq p \leq+\infty$, the space similarly defined (with $d v(x) d \gamma(y)$ in the integrand).

Proposition 2.2. (i) The Fourier-Wigner transform $V$ is a bilinear mapping from $D_{*}(\mathbb{R}) \times D_{*}(\mathbb{R})$ into $S_{*}\left(\mathbb{R}^{2}\right)$.

(ii) For $p \in] 1,2]$ and $p^{\prime}$ such that $1 / p+1 / p^{\prime}=1$, we have

$$
\|V(f, g)\|_{p^{\prime}, \nu \otimes \gamma} \leq\|f\|_{p, v}\|g\|_{p^{\prime}, v} .
$$

The transform $V$ can be extended to a continuous bilinear operator, denoted also by $V$, from $L^{p}(d \nu) \times L^{p^{\prime}}(d \nu)$ into $L^{p^{\prime}}(d \nu \otimes d \gamma)$.

Proof. (i) Let $F$ be the function defined on $\mathbb{R}^{2}$ by $F(x, y)=f(y) \mathscr{T}_{x} g(y)$. It's clear that $F \in D_{*}\left(\mathbb{R}^{2}\right)$, and we have

$$
V(f, g)(x, \lambda)=I \otimes \mathscr{F}(F)(x, \lambda),
$$

where $I$ is the identity operator. This and the fact that $\mathscr{F}$ is a topological isomorphism from $D_{*}(\mathbb{R})$ onto $\mathbb{H}_{*}(\mathbb{C})$ imply (i).

(ii) This follows from the first equality in (2-1) together with Proposition 1.3, Minkowski's inequality for integrals [Folland 1984, p.186], and the fact that

$$
\left\|\mathscr{T}_{x} g\right\|_{p^{\prime}, v} \leq\|g\|_{p^{\prime}, v} \quad \text { for } x \in \mathbb{R} .
$$

Theorem 2.3. For $f, g \in D_{*}(\mathbb{R})$, we have

$$
\mathscr{F} \otimes \mathscr{F}^{-1}(V(f, g))(\mu, \lambda)=\varphi_{\mu}(\lambda) f(\lambda) \mathscr{F} g(\mu) \quad \text { for } \mu, \lambda \in \mathbb{R} .
$$

Proof. Using Definition 2.1 and Fubini's Theorem we have, for all $\mu, \lambda \in \mathbb{R}$,

$$
\begin{aligned}
\mathscr{F} \otimes \mathscr{F}^{-1}(V(f, g))(\mu, \lambda) & =\int_{0}^{+\infty} \int_{0}^{+\infty} V(f, g)(x, y) \varphi_{\mu}(x) \varphi_{y}(\lambda) d v(x) d \gamma(y) \\
& =\int_{0}^{+\infty} \int_{0}^{+\infty} \mathscr{F}\left(f \mathscr{T}_{x} g\right)(y) \varphi_{\mu}(x) \varphi_{y}(\lambda) d v(x) d \gamma(y) \\
& =\int_{0}^{+\infty} \varphi_{\mu}(x)\left(\int_{0}^{+\infty} \mathscr{F}\left(f \mathscr{T}_{x} g\right)(y) \varphi_{y}(\lambda) d \gamma(y)\right) d v(x) .
\end{aligned}
$$


From (1-8) we deduce

$$
\begin{aligned}
\mathscr{F}_{F} \otimes \mathscr{F}^{-1}(V(f, g))(\mu, \lambda) & =\int_{0}^{+\infty} \varphi_{\mu}(x) f(\lambda) \mathscr{T}_{x} g(\lambda) d v(x) \\
& =f(\lambda) \mathscr{F}\left(\mathscr{T}_{\lambda} g\right)(\mu)=f(\lambda) \varphi_{\mu}(\lambda) \mathscr{F} g(\mu) .
\end{aligned}
$$

Corollary 2.4. For all $f, g \in D_{*}(\mathbb{R})$, we have

$$
\begin{array}{ll}
\int_{0}^{+\infty} \mathscr{F} \otimes \mathscr{F}^{-1}(V(f, g))(\mu, \lambda) d v(\lambda)=\mathscr{F} f(\mu) \mathscr{F} g(\mu) & \text { for } \mu \in \mathbb{R}, \\
\int_{0}^{+\infty} \mathscr{F} \otimes \mathscr{F}^{-1}(V(f, g))(\mu, \lambda) d \gamma(\mu)=f(\lambda) g(\lambda) & \text { for } \lambda \in \mathbb{R} .
\end{array}
$$

Proof. Theorem 2.3 gives

$$
\begin{aligned}
\int_{0}^{+\infty} \mathscr{F} \otimes \mathscr{F}^{-1}(V(f, g))(\mu, \lambda) d \nu(\lambda) & =\int_{0}^{+\infty} \varphi_{\mu}(\lambda) f(\lambda) \mathscr{F} g(\mu) d \nu(\lambda) \\
& =\mathscr{F} f(\mu) \mathscr{F} g(\mu) \quad \text { for } \mu \in \mathbb{R}, \\
\int_{0}^{+\infty} \mathscr{F} \otimes \mathscr{F}^{-1}(V(f, g))(\mu, \lambda) d \gamma(\mu) & =\int_{0}^{+\infty} \varphi_{\mu}(\lambda) f(\lambda) \mathscr{F} g(\mu) d \gamma(\mu) \\
& =f(\lambda) \int_{0}^{+\infty} \varphi_{\mu}(\lambda) \mathscr{F} g(\mu) d \gamma(\mu) \\
& =f(\lambda) g(\lambda) \text { for } \lambda \in \mathbb{R} .
\end{aligned}
$$

Theorem 2.5. Let $f, g \in L^{1}(d v) \cap L^{2}(d v)$ be such that $c=\int_{0}^{+\infty} g(x) d v(x) \neq 0$. Then

$$
\mathscr{F} f(\lambda)=\frac{1}{c} \int_{0}^{+\infty} V(f, g)(x, \lambda) d \nu(x) \quad \text { for } \lambda \in \mathbb{R} .
$$

Proof. From Definition 2.1, we have

$$
\int_{0}^{+\infty} V(f, g)(x, \lambda) d v(x)=\int_{0}^{+\infty}\left(\int_{0}^{+\infty} f(y) \mathscr{T}_{x} g(y) \varphi_{\lambda}(y) d v(y)\right) d v(x)
$$

for all $\lambda \in \mathbb{R}$. The result follows from Fubini's Theorem and the equality

$$
\int_{0}^{+\infty} \mathscr{T}_{x} g(y) d v(y)=\int_{0}^{+\infty} g(x) d v(x)=c .
$$

Corollary 2.6. With the hypothesis of Theorem 2.5, if $\mathscr{F} f \in L^{1}(d \gamma)$, we have the following inversion formula for the Fourier-Wigner transform $V$ :

$$
f(x)=\frac{1}{c} \int_{0}^{+\infty} \varphi_{\mu}(x)\left(\int_{0}^{+\infty} V(f, g)(y, \mu) d \nu(y)\right) d \gamma(\mu) \quad \text { for a.e. } x \in \mathbb{R} .
$$




\section{The Weyl transform associated with $\Delta$}

We now introduce the Weyl transform and relate it to the Fourier-Wigner transform. To do this, we must define the class of pseudodifferential operators [Wong 1998].

Definition 3.1. Let $m \in \mathbb{R}$. We define $S^{m}$ to be the set of all infinitely differentiable functions $\sigma$ on $\mathbb{R} \times \mathbb{R}$, even with respect to each variable, and such that for all $p, q \in \mathbb{N}$, there exists a positive constant $C_{p, q, m}$ satisfying

$$
\left|\left(\frac{\partial}{\partial x}\right)^{p}\left(\frac{\partial}{\partial y}\right)^{q} \sigma(x, y)\right| \leq C_{p, q, m}\left(1+y^{2}\right)^{m-q} .
$$

Definition 3.2. For $m \in \mathbb{R}$ and $\sigma \in S^{m}$, we define the operator $H_{\sigma}$ on $D_{*}(\mathbb{R}) \times$ $D_{*}(\mathbb{R})$ by

$$
H_{\sigma}(f, g)(\lambda)=\int_{0}^{+\infty}\left(\int_{0}^{+\infty} \sigma(x, y) \varphi_{y}(\lambda) V(f, g)(x, y) d v(x)\right) d \gamma(y),
$$

for all $\lambda \in \mathbb{R}$, and we put

$$
\mathbb{H}_{\sigma}(f, g)=H_{\sigma}(f, g)(0) .
$$

Proposition 3.3. Define $\sigma \in S^{m}$ by $\sigma(x, y)=-y^{2}$ for $x, y \in \mathbb{R}$. Then, for all $f, g \in D_{*}(\mathbb{R})$, we have

$$
H_{\sigma}(f, g)(\lambda)=c \Delta f(\lambda) \quad \text { for } \lambda \in \mathbb{R}
$$

where $c=\int_{0}^{+\infty} g(x) d v(x)$.

Proof. From (3-1), we have

$$
H_{\sigma}(f, g)(\lambda)=\int_{0}^{+\infty}\left(\int_{0}^{+\infty}-y^{2} \varphi_{y}(\lambda) V(f, g)(x, y) d \nu(x)\right) d \gamma(y) \text { for } \lambda \in \mathbb{R} .
$$

Using Definition 2.1 we obtain

$$
H_{\sigma}(f, g)(\lambda)=\int_{0}^{+\infty}\left(\int_{0}^{+\infty}-y^{2} \varphi_{y}(\lambda)\left(\int_{0}^{+\infty} f(z) \mathscr{T}_{x} g(z) \varphi_{y}(z) d v(z)\right) d \nu(x)\right) d \gamma(y)
$$

for $\lambda \in \mathbb{R}$. From Fubini's Theorem, we get

$$
\begin{aligned}
H_{\sigma}(f, g)(\lambda) & \\
& =\int_{0}^{+\infty}-y^{2} \varphi_{y}(\lambda)\left(\int_{0}^{+\infty} f(z) \varphi_{y}(z)\left(\int_{0}^{+\infty} \mathscr{T}_{z} g(x) d \nu(x)\right) d \nu(z)\right) d \gamma(y) \\
& =c \int_{0}^{+\infty}-y^{2} \varphi_{y}(\lambda)\left(\int_{0}^{+\infty} f(z) \varphi_{y}(z) d \nu(z)\right) d \gamma(y) \\
& =c \int_{0}^{+\infty}-y^{2} \varphi_{y}(\lambda) \mathscr{F} f(y) d \gamma(y) .
\end{aligned}
$$


But, for all $y \in \mathbb{R},-y^{2} \mathscr{F} f(y)=\mathscr{F}(\Delta f)(y)$. We complete the proof using the inversion formula (1-8).

Definition 3.4. Let $\sigma \in S^{m} ; m<-\alpha-1$. The Weyl transform associated with $\Delta$ is the mapping $W_{\sigma}$ defined on $D_{*}(\mathbb{R})$ by

$$
W_{\sigma}(f)(\lambda)=\int_{0}^{+\infty}\left(\int_{0}^{+\infty} \varphi_{y}(\lambda) \sigma(x, y) \mathscr{T}_{\lambda} f(x) d \nu(x)\right) d \gamma(y) \quad \text { for } \lambda \in \mathbb{R} .
$$

Notation. We denote by

- $S_{*}(\mathbb{R})$ the space of even, infinitely differentiable functions on $\mathbb{R}$, rapidly decreasing together with all their derivatives.

- $S_{*}^{2}(\mathbb{R})=\varphi_{0} S_{*}(\mathbb{R})$, where $\varphi_{0}$ is the solution of $(1-2)$ with $\lambda=0$.

For $\rho=0$ these two spaces coincide [Trimèche 1997]. The Fourier transform $\mathscr{F}$ is a topological isomorphism from $S_{*}^{2}(\mathbb{R})$ onto $S_{*}(\mathbb{R})$, whose inverse is given by (1-12).

Lemma 3.5. For $\sigma \in D_{*}\left(\mathbb{R}^{2}\right)$, the function $k$ defined by

$$
k(x, y)=\int_{0}^{+\infty} \varphi_{\lambda}(x) \mathscr{T}_{x}(\sigma(\cdot, \lambda))(y) d \gamma(\lambda) \quad \text { for } x, y \in \mathbb{R}
$$

belongs to $L^{p}(d v \otimes d v)$, for all $p \in[2,+\infty[$.

Proof. The defining equation of $k$ can be rewritten $k(x, y)=\mathscr{T}_{x}(G(\cdot, x))(y)$, where

$$
G(x, y)=I \otimes \mathscr{F}^{-1}(\sigma)(x, y) \text { for } x, y \in \mathbb{R},
$$

for $I$ the identity operator. It follows that, for all $p \in[2,+\infty[$,

$$
\begin{aligned}
\int_{0}^{+\infty} \int_{0}^{+\infty}|k(x, y)|^{p} d v(x) d v(y) & =\int_{0}^{+\infty}\left(\int_{0}^{+\infty}\left|\mathscr{T}_{x}(G(\cdot, x)(y))\right|^{p} d v(y)\right) d v(x) \\
& \leq \int_{0}^{+\infty}\left(\int_{0}^{+\infty}|G(y, x)|^{p} d v(y)\right) d v(x) \\
& \leq \int_{0}^{+\infty}\left(\int_{0}^{+\infty}\left|I \otimes \mathscr{F}^{-1}(\sigma)(y, x)\right|^{p} d v(y)\right) d v(x) .
\end{aligned}
$$

We distinguish two cases, $p=2$ and $p \in] 2,+\infty[$, the case $p=+\infty$ being trivial. For $p=2$,

$$
\int_{0}^{+\infty} \int_{0}^{+\infty}|k(x, y)|^{2} d v(x) d v(y) \leq \int_{0}^{+\infty}\left(\int_{0}^{+\infty}\left|\mathscr{F}^{-1}(\sigma(x, \cdot)(y))\right|^{2} d v(x)\right) d v(y) .
$$

From (1-10) we deduce that

$$
\int_{0}^{+\infty} \int_{0}^{+\infty}|k(x, y)|^{2} d \nu(x) d \nu(y) \leq \int_{0}^{+\infty}\left(\int_{0}^{+\infty}|\sigma(y, x)|^{2} d \gamma(y)\right) d \nu(y)<+\infty,
$$


because $\sigma$ belongs to $D_{*}\left(\mathbb{R}^{2}\right)$. The case $\left.p \in\right] 2,+\infty[$ is more complex. From the hypotheses on $\Delta$, we deduce that, as $x \rightarrow+\infty$,

$$
A(x) \sim \begin{cases}x^{2 \alpha+1} & \text { if } \rho=0, \\ \exp (2 \rho x) & \text { if } \rho>0 .\end{cases}
$$

- For $\rho=0$, recall that $\mathscr{F}$ is an isomorphism from $S_{*}(\mathbb{R})$ onto itself. Thus $I \otimes \mathscr{F}^{-1}(\sigma)$ belongs to $S_{*}\left(\mathbb{R}^{2}\right)$, and the asymptotics (3-3) implies

$$
\begin{aligned}
& \int_{0}^{+\infty} \int_{0}^{+\infty}|k(x, y)|^{p} d \nu(x) d v(y) \\
& \leq \int_{0}^{+\infty}\left(\int_{0}^{+\infty}\left|I \otimes \mathscr{F}^{-1}(\sigma)(y, x)\right|^{p} d v(x)\right) d v(y)<+\infty
\end{aligned}
$$

- For $\rho>0$, we have from [Trimèche 1997, p. 99]

$$
\left|\varphi_{\lambda}(x)\right| \leq \varphi_{0}(x) \leq m(1+x) \exp (-\rho x) \quad \text { for all } \lambda \in \mathbb{R} \text { and } x \geq 0,
$$

where $m$ is a positive constant. Then

$$
\left|I \otimes \mathscr{F}^{-1}(\sigma)(y, x)\right| \leq m(1+x) \exp (-\rho x) \int_{0}^{+\infty}|\sigma(y, z)| d v(z) .
$$

Since $\sigma$ belongs to $D_{*}\left(\mathbb{R}^{2}\right)$, there exists a positive constant $M$ such that

$$
\int_{0}^{+\infty}|\sigma(y, z)| d \nu(z) \leq M \quad \text { for } y \geq 0,
$$

which implies that

$$
\left|I \otimes \mathscr{F}^{-1}(\sigma)(y, x)\right| \leq m M(1+x) \exp (-\rho x) .
$$

This, together with the asymptotics (3-3), implies the validity of the same bound (3-4) as in the previous case.

Theorem 3.6. Let $\sigma \in D_{*}\left(\mathbb{R}^{2}\right)$ and $f \in D_{*}(\mathbb{R})$.

(i) $W_{\sigma}(f)(x)=\int_{0}^{+\infty} k(x, y) f(y) d v(y)$ for all $x \in \mathbb{R}$.

(ii) $\left\|W_{\sigma}(f)\right\|_{p^{\prime}, v} \leq\|k\|_{p^{\prime}, v \otimes v}\|f\|_{p, v}$ for $p \in[1,2]$ and $p^{\prime}$ such that $1 / p+1 / p^{\prime}=1$.

(iii) $W_{\sigma}$ can be extended to a bounded operator from $L^{p}(d v)$ into $L^{p^{\prime}}(d v)$. In particular, $W_{\sigma}: L^{2}(d v) \rightarrow L^{2}(d v)$ is a Hilbert-Schmidt operator, hence compact. 
Proof. (i) From Definition 3.4, we have, for all $x \in \mathbb{R}$;

$$
\begin{aligned}
W_{\sigma}(f)(x) & =\int_{0}^{+\infty} \varphi_{y}(x)\left(\int_{0}^{+\infty} \sigma(z, y) \mathscr{T}_{x} f(z) d \nu(z)\right) d \gamma(y) \\
& =\int_{0}^{+\infty} \varphi_{y}(x)\left(\int_{0}^{+\infty} f(z) \mathscr{T}_{x}[\sigma(., y)](z) d \nu(z)\right) d \gamma(y)
\end{aligned}
$$

From Fubini's Theorem, we get, for all $x \in \mathbb{R}$,

$$
\begin{aligned}
W_{\sigma}(f)(x) & =\int_{0}^{+\infty} f(z)\left(\int_{0}^{+\infty} \varphi_{y}(x) \mathscr{T}_{x}[\sigma(., y)](z) d \gamma(y)\right) d \nu(z) \\
& =\int_{0}^{+\infty} f(z) k(x, z) d \nu(z) .
\end{aligned}
$$

(ii) Follows from (i), Hölder's inequality, and Lemma 3.5.

(iii) Since $k \in L^{2}(d v \otimes d v)$, the mapping

$$
W_{\sigma}: L^{2}(d v) \longrightarrow L^{2}(d v)
$$

is a Hilbert-Schmidt operator, and so compact.

Theorem 3.7. Let $m<-\alpha-1$ and $\sigma \in S^{m}$. For all $f, g \in D_{*}(\mathbb{R})$,

$$
\mathbb{H}_{\sigma}(f, g)=\int_{0}^{+\infty} f(x) W_{\sigma} g(x) d \nu(x) .
$$

Proof. Using (3-2) and Definition 2.1 we obtain

$$
\begin{aligned}
\mathbb{H}_{\sigma}(f, g) & =\int_{0}^{+\infty}\left(\int_{0}^{+\infty} \sigma(x, y) V(f, g)(x, y) d v(x)\right) d \gamma(y) \\
& =\int_{0}^{+\infty}\left(\int_{0}^{+\infty} \sigma(x, y)\left(\int_{0}^{+\infty} f(\lambda) \mathscr{T}_{x} g(\lambda) \varphi_{y}(\lambda) d \nu(\lambda)\right) d \nu(x)\right) d \gamma(y) .
\end{aligned}
$$

From Fubini's theorem, we get

$$
\begin{aligned}
\mathbb{H}_{\sigma}(f, g) & =\int_{0}^{+\infty} f(\lambda)\left(\int_{0}^{+\infty} \varphi_{y}(\lambda)\left(\int_{0}^{+\infty} \sigma(x, y) \mathscr{T}_{x} g(\lambda) d \nu(x)\right) d \gamma(y)\right) d \nu(\lambda) \\
& =\int_{0}^{+\infty} f(\lambda) W_{\sigma}(g)(\lambda) d \nu(\lambda)
\end{aligned}
$$

4. The Weyl transform with symbol in $L^{p}(d v \otimes d \gamma)$, for $1 \leq p \leq 2$

In this section we show using (3-5) that, if $1 \leq p \leq 2$, the Weyl transform with symbol in $L^{p}(d v \otimes d \gamma)$ is a compact operator. 
Notation. We denote by $\mathscr{B}\left(L^{2}(d v)\right)$ the $\mathbb{C}^{*}$-algebra of bounded operators $\Psi$ from $L^{2}(d v)$ into itself, equipped with the norm

$$
\|\Psi\|_{*}=\sup _{\|f\|_{2, v}=1}\|\Psi(f)\|_{2, v} .
$$

Theorem 4.1. Let $\langle\cdot / \cdot\rangle$ denote the inner product in $L^{2}(d v)$. There exists a unique operator $Q: L^{2}(d v \otimes d \gamma) \rightarrow \mathscr{B}\left(L^{2}(d v)\right)$, whose action we denote by $\sigma \mapsto Q_{\sigma}$, such that

$$
\left\langle Q_{\sigma}(g) / \bar{f}\right\rangle=\int_{0}^{+\infty}\left(\int_{0}^{+\infty} \sigma(x, y) V(f, g)(x, y) d v(x)\right) d \gamma(y) \quad \text { for } f, g \in L^{2}(d v) .
$$

Furthermore, $\left\|Q_{\sigma}\right\|_{*} \leq\|\sigma\|_{2, \nu \otimes \gamma}$.

Proof. Let $\sigma \in D_{*}\left(\mathbb{R}^{2}\right)$. For $g \in D_{*}(\mathbb{R})$, put $Q_{\sigma}(g)=W_{\sigma}(g)$. From Theorems 3.6 and 3.7, we obtain

$$
\begin{aligned}
\left\langle Q_{\sigma}(g) / \bar{f}\right\rangle & =\left\langle W_{\sigma}(g) / \bar{f}\right\rangle=\mathbb{H}_{\sigma}(f, g) \\
& =\int_{0}^{+\infty}\left(\int_{0}^{+\infty} \sigma(x, y) V(f, g)(x, y) d \nu(x)\right) d \gamma(y) .
\end{aligned}
$$

On the other hand, from Proposition 2.2(ii), we have

$$
\left|\left\langle Q_{\sigma}(g) / \bar{f}\right\rangle\right| \leq\|\sigma\|_{2, \nu \otimes \gamma}\|f\|_{2, v}\|g\|_{2, v} .
$$

Thus $Q_{\sigma} \in \mathscr{B}\left(L^{2}(d v)\right)$ and

$$
\left\|Q_{\sigma}\right\|_{*} \leq\|\sigma\|_{2, \nu \otimes \gamma} .
$$

Now consider $\sigma \in L^{2}(d v \otimes d \gamma)$. Let $\left(\sigma_{k}\right)_{k \in \mathbb{N}}$ be a sequence in $D_{*}\left(\mathbb{R}^{2}\right)$ such that $\left\|\sigma_{k}-\sigma\right\|_{2, \nu \otimes \gamma}$ approaches 0 as $k \rightarrow+\infty$. From (4-1) we have, for all $k, l \in \mathbb{N}$,

$$
\left\|Q_{\sigma_{k}}-Q_{\sigma_{l}}\right\|_{*} \leq\left\|\sigma_{k}-\sigma_{l}\right\|_{2, \nu \otimes \gamma} \leq\left\|\sigma_{k}-\sigma\right\|_{2, \nu \otimes \gamma}+\left\|\sigma_{l}-\sigma\right\|_{2, \nu \otimes \gamma} .
$$

Thus $\left(Q_{\sigma_{k}}\right)_{k \in \mathbb{N}}$ is a Cauchy sequence in $\mathscr{B}\left(L^{2}(d v)\right)$. Let it converge to $Q_{\sigma}$. Clearly $Q_{\sigma}$ is independent from the choice of $\left(\sigma_{k}\right)_{k \in \mathbb{N}}$, and we have

$$
\left\|Q_{\sigma}\right\|_{*}=\lim _{k \rightarrow+\infty}\left\|Q_{\sigma_{k}}\right\|_{*} \leq \lim _{k \rightarrow+\infty}\left\|\sigma_{k}\right\|_{2, \nu \otimes \gamma}=\|\sigma\|_{2, \nu \otimes \gamma} .
$$

We consider first $f, g \in D_{*}(\mathbb{R})$. Then

$$
\begin{aligned}
\left\langle Q_{\sigma}(g) / \bar{f}\right\rangle & =\lim _{k \rightarrow+\infty}\left\langle Q_{\sigma_{k}}(g) / \bar{f}\right\rangle \\
& =\lim _{k \rightarrow+\infty} \int_{0}^{+\infty}\left(\int_{0}^{+\infty} \sigma_{k}(x, y) V(f, g)(x, y) d \nu(x)\right) d \gamma(y) \\
& =\int_{0}^{+\infty}\left(\int_{0}^{+\infty} \sigma(x, y) V(f, g)(x, y) d \nu(x)\right) d \gamma(y) .
\end{aligned}
$$


Now let $f, g$ be in $L^{2}(d v)$. Pick sequences $\left(f_{k}\right)_{k \in \mathbb{N}}$, and $\left(g_{k}\right)_{k \in \mathbb{N}}$ in $D_{*}(\mathbb{R})$ converging to $f$ and $g$, respectively, in the $\|\cdot\|_{2, v}$-norm. Then

$$
\begin{aligned}
\left\langle Q_{\sigma}(g) / \bar{f}\right\rangle & =\lim _{k \rightarrow+\infty}\left\langle Q_{\sigma}\left(g_{k}\right) / \bar{f}_{k}\right\rangle \\
& =\lim _{k \rightarrow+\infty} \int_{0}^{+\infty}\left(\int_{0}^{+\infty} \sigma(x, y) V\left(f_{k}, g_{k}\right)(x, y) d v(x)\right) d \gamma(y) \\
& =\int_{0}^{+\infty}\left(\int_{0}^{+\infty} \sigma(x, y) V(f, g)(x, y) d v(x)\right) d \gamma(y) .
\end{aligned}
$$

We now give an extension of Theorem 4.1 that will allow us to prove that for $1 \leq p \leq 2$ the Weyl transform with symbol in $L^{p}(d \nu \otimes d \gamma)$, is a compact operator.

Theorem 4.2. Let $p \in[1,2]$. There exists a unique bounded operator

$$
Q: L^{p}(d v \otimes d \gamma) \rightarrow \mathscr{B}\left(L^{2}(d v)\right),
$$

whose action is denoted by $\sigma \rightarrow Q_{\sigma}$, such that

$$
\left\langle Q_{\sigma}(g) / \bar{f}\right\rangle=\int_{0}^{+\infty}\left(\int_{0}^{+\infty} \sigma(x, y) V(f, g)(x, y) d \nu(x)\right) d \gamma(y) \quad \text { for } f, g \in D_{*}(\mathbb{R}) .
$$

Moreover, $\left\|Q_{\sigma}\right\|_{*} \leq\|\sigma\|_{p, \nu \otimes \gamma}$.

Proof. The case $p=2$ is given by Theorem 4.1. We turn to the case $p=1$. For $\sigma \in D_{*}\left(\mathbb{R}^{2}\right)$, we define $Q_{\sigma}$ by

$$
Q_{\sigma}(g)=W_{\sigma}(g) \quad \text { for } g \in D_{*}(\mathbb{R}) .
$$

From Theorems 3.6 and 3.7, we have, for $f \in D_{*}(\mathbb{R})$,

$$
\left\langle Q_{\sigma}(g) / \bar{f}\right\rangle=\mathbb{H}_{\sigma}(f, g)=\int_{0}^{+\infty}\left(\int_{0}^{+\infty} \sigma(x, y) V(f, g)(x, y) d \nu(x)\right) d \gamma(y) .
$$

From Hölder's inequality we then obtain

$$
\left|\left\langle Q_{\sigma}(g) / \bar{f}\right\rangle\right| \leq\|\sigma\|_{1, \nu \otimes \gamma}\|V(f, g)\|_{\infty, v \otimes \gamma} \leq\|\sigma\|_{1, \nu \otimes \gamma}\|f\|_{2, v}\|g\|_{2, v} .
$$

This shows that $Q_{\sigma} \in \mathscr{B}\left(L^{2}(d \nu)\right)$ and $\left\|Q_{\sigma}\right\|_{*} \leq\|\sigma\|_{1, \nu \otimes \gamma}$.

We extend the definition of $Q_{\sigma}$ and the two facts just proved to the case of $\sigma \in L^{1}(d \nu \otimes d \gamma)$, working as in the proof of Theorem 4.1.

Finally, the Riesz-Thorin Theorem [Stein 1956; Stein and Weiss 1971], allows us to generalize the same results from the cases $p=1$ and $p=2$ to all $p \in[1,2]$.

Theorem 4.3. Let $p \in[1,2]$. For $\sigma \in L^{p}(d v \otimes d \gamma)$, the operator $Q_{\sigma}$ from $L^{2}(d v)$ into itself is compact. 
Proof. Given $\sigma \in L^{p}(d v \otimes d \gamma)$, choose a sequence $\left(\sigma_{k}\right)_{k \in \mathbb{N}}$ in $D_{*}\left(\mathbb{R}^{2}\right)$ approximating $\sigma$ in the $\|\cdot\|_{p, v \otimes \gamma}$-norm. The last assertion of Theorem 4.2 says that

$$
\left\|Q_{\sigma_{k}}-Q_{\sigma}\right\|_{*} \leq\left\|\sigma_{k}-\sigma\right\|_{p, \nu \otimes \gamma}
$$

so $Q_{\sigma_{k}}$ approaches $Q_{\sigma}$ in $\mathscr{B}\left(L^{2}(d v)\right)$. From Theorem 3.6 we know that $W_{\sigma_{k}}=$ $Q_{\sigma_{k}}$ is compact for all $k \in \mathbb{N}$. The theorem then follows from the fact that the subspace $\mathscr{K}\left(L^{2}(d v)\right)$ of $\mathscr{B}\left(L^{2}(d v)\right)$ consisting of compact operators is a closed ideal of $\mathscr{B}\left(L^{2}(d v)\right)$.

\section{The Weyl transform with symbol in $S_{*, 0}^{\prime}\left(\mathbb{R}^{2}\right)$}

Notation. We denote by

- $S_{*, 0}\left(\mathbb{R}^{2}\right)$ the subspace of $S_{*}\left(\mathbb{R}^{2}\right)$ consisting of functions with compact support with respect to the first variable;

- $S_{*, 0}^{\prime}\left(\mathbb{R}^{2}\right)$ the topological dual of $S_{*, 0}\left(\mathbb{R}^{2}\right)$;

- $D_{*}^{\prime}(\mathbb{R})$ the space of even distribution on $\mathbb{R}$. It is the topological dual of $D_{*}(\mathbb{R})$.

Definition 5.1. For $\sigma \in S_{*, 0}^{\prime}\left(\mathbb{R}^{2}\right)$ and $g \in D_{*}(\mathbb{R})$, we define the operator $W_{\sigma}(g)$ on $D_{*}(\mathbb{R})$ by

$$
\left(W_{\sigma}(g)\right)(f)=\sigma(V(f, g)) \text { for } f \in D_{*}(\mathbb{R}),
$$

where $V$ is the mapping from Definition 2.1. Clearly $W_{\sigma}(g)$ belongs to $D_{*}^{\prime}(\mathbb{R})$.

Proposition 5.2. Consider the distribution $\sigma$ of $S_{*, 0}^{\prime}\left(\mathbb{R}^{2}\right)$ given by the constant function 1 . For all $g \in D_{*}(\mathbb{R})$, we have

$$
W_{\sigma}(g)=c \delta,
$$

where $c=\int_{0}^{+\infty} g(x) d v(x)$ and $\delta$ is the Dirac distribution at 0.

Proof. For $f, g \in D_{*}(\mathbb{R})$, we get

$$
\left(W_{\sigma}(g)\right)(f)=\sigma(V(f, g))=\int_{0}^{+\infty}\left(\int_{0}^{+\infty} V(f, g)(x, y) d \nu(x)\right) d \gamma(y) .
$$

But from the proof of Theorem 2.5, we have

$$
\int_{0}^{+\infty} V(f, g)(x, y) d v(x)=c \mathscr{F} f(y) \quad \text { for } y \in \mathbb{R} .
$$

Integrating both sides over $[0,+\infty$ [ with respect to the measure $d \gamma$ and using (1-8), we obtain

$$
\sigma(V(f, g))=\left(W_{\sigma}(g)\right)(f)=c \int_{0}^{+\infty} \mathscr{F} f(y) d \gamma(y)=c f(0)=(c \delta, f) .
$$


Note that by Proposition 5.2, there exists $\sigma \in S_{*, 0}^{\prime}\left(\mathbb{R}^{2}\right)$, given by a function in $L^{\infty}(d v \otimes d \gamma)$, such that for all $g \in D_{*}(\mathbb{R})$ satisfying $c=\int_{0}^{+\infty} g(x) d \nu(x) \neq 0$, the distribution $W_{\sigma}(g)$ is not given by a function in $L^{2}(d v)$.

\section{The Weyl transform with symbol in $L^{p}(d v \otimes d \gamma)$, for $2<p<\infty$}

Theorem 6.1. Let $p \in] 2, \infty\left[\right.$. There exists a function $\sigma \in L^{p}(d v \otimes d \gamma)$ such that the Weyl transform $W_{\sigma}$ defined by $(5-1)$ is not a bounded linear operator on $L^{2}(d v)$.

We break down the proof into two lemmas, of which the theorem is an immediate consequence.

Lemma 6.2. Let $p \in] 2, \infty\left[\right.$. Suppose that for all $\sigma \in L^{p}(d v \otimes d \gamma)$, the Weyl transform $W_{\sigma}$ given by (5-1) is a bounded linear operator on $L^{2}(d \nu)$. Then there exists a positive constant $M$ such that

$$
\left\|W_{\sigma}\right\|_{*} \leq M\|\sigma\|_{p, \nu \otimes \gamma} \quad \text { for all } \sigma \in L^{p}(d \nu \otimes d \gamma) .
$$

Proof. Under the assumption of the lemma, there exists for each $\sigma \in L^{p}(d v \otimes d \gamma)$ a positive constant $C_{\sigma}$ such that

$$
\left\|W_{\sigma}(g)\right\|_{2, \nu} \leq C_{\sigma}\|g\|_{2, v} \quad \text { for } g \in L^{2}(d v) .
$$

Let $f, g \in D_{*}(\mathbb{R})$ be such that $\|f\|_{2, v}=\|g\|_{2, v}=1$ and define a linear operator $Q_{f, g}: L^{p}(d v \otimes d \gamma) \rightarrow \mathbb{C}$ by

$$
Q_{f, g}(\sigma)=\left\langle W_{\sigma}(g) / \bar{f}\right\rangle .
$$

Then

$$
\sup _{|f|_{2, v}=|g|_{2, v}=1}\left|Q_{f, g}(\sigma)\right| \leq C_{\sigma} .
$$

By the Banach-Steinhaus theorem, the operator $Q_{f, g}$ is bounded on $L^{p}(d \nu \otimes d \gamma)$, so there exists $M>0$ such that

$$
\left\|Q_{f, g}\right\|=\sup _{\|\sigma\|_{p, \nu \otimes \gamma}=1}\left|Q_{f, g}(\sigma)\right| \leq M .
$$

From this we deduce that for all $f, g \in D_{*}(\mathbb{R})$ and $\sigma \in L^{p}(d \nu \otimes d \gamma)$,

$$
\left|\left\langle W_{\sigma}(g) / \bar{f}\right\rangle\right| \leq M\|\sigma\|_{p, \nu \otimes \gamma}\|f\|_{2, v}\|g\|_{2, \nu},
$$

which implies (6-1).

Lemma 6.3. For $2<p<\infty$, there is no positive constant $M$ satisfying (6-1). 
Proof. Suppose there exists such an $M$. Let $p^{\prime}$ be such that $1 / p+1 / p^{\prime}=1$. Then $\left.p^{\prime} \in\right] 1,2\left[\right.$. We consider, for $f, g \in D_{*}(\mathbb{R})$, the function $V(f, g)$ of Definition 2.1. We have

$$
\begin{aligned}
\|V(f, g)\|_{p^{\prime}, \nu \otimes \gamma} & =\sup _{\|\sigma\|_{p, \nu \otimes \gamma}=1}\left|\int_{0}^{+\infty} \int_{0}^{+\infty} \sigma(x, y) V(f, g)(x, y) d \nu(x) d \gamma(y)\right| \\
& =\sup _{\|\sigma\|_{p, \nu \otimes \gamma}=1}\left|\left\langle W_{\sigma}(g) / \bar{f}\right\rangle\right| \leq \sup _{\|\sigma\|_{p, \nu \otimes \gamma}=1}\left\|W_{\sigma}(g)\right\|_{2, \nu}\|f\|_{2, \nu},
\end{aligned}
$$

and consequently

$$
\|V(f, g)\|_{p^{\prime}, v \otimes \gamma} \leq M\|f\|_{2, v}\|g\|_{2, v} .
$$

Now consider $f, g$ in $L^{2}(d v)$. Choose sequences $\left(f_{k}\right)_{k \in \mathbb{N}}$ and $\left(g_{k}\right)_{k \in \mathbb{N}}$ in $D_{*}(\mathbb{R})$ approximating $f$ and $g$ in the $\|\cdot\|_{2, v}$-norm. By Proposition 2.2, the sequence $\left(V\left(f_{k}, g_{k}\right)\right)_{k \in \mathbb{N}}$ converges to $V(f, g)$ in $L^{p^{\prime}}(d v \otimes d \gamma)$, and thus we have extended (6-2) to all $f, g \in L^{2}(d \nu)$. We will exhibit an example where this leads to a contradiction.

Let $f$ be an even, measurable function on $\mathbb{R}$, supported in $[-1,1]$. We have

$$
|V(f, f)(x, y)| \leq|f| *|f|(x),
$$

where $*$ is the convolution product (Definition 1.1). From (1-7), we deduce that for all $y \in \mathbb{R}$, the function $x \mapsto V(f, f)(x, y)$ is supported in [-2,2]. Hölder's inequality gives

$$
\begin{aligned}
\left(\int_{0}^{+\infty}\left|\int_{0}^{2} V(f, f)(x, y) d v(x)\right|^{p^{\prime}} d \gamma(y)\right)^{1 / p^{\prime}} & \\
& \leq\left(\int_{0}^{2} d v(x)\right)^{1 / p}\left(\int_{0}^{+\infty}\left(\int_{0}^{2}|V(f, f)(x, y)|^{p^{\prime}} d v(x)\right) d \gamma(y)\right)^{1 / p^{\prime}} \\
& =\left(\int_{0}^{2} d v(x)\right)^{1 / p}\|V(f, f)\|_{p^{\prime}, v \otimes \gamma} \leq M\left(\int_{0}^{2} d v(x)\right)^{1 / p}\|f\|_{2, v}^{2},
\end{aligned}
$$

the last inequality following from (6-2). This proves that the function

$$
y \mapsto \int_{0}^{+\infty} V(f, f)(x, y) d v(x)=c \mathscr{F} f(y)
$$

belongs to $L^{p^{\prime}}(d \gamma)$; here $c=\int_{0}^{+\infty} f(x) d v(x)$. and we have used the proof of Theorem 2.5 for the equality on the right-hand side. Putting this together with the preceding inequality we see that, if $c \neq 0$, the function $\mathscr{F} f$ belongs to $L^{p^{\prime}}(d \gamma)$ and

$$
\|\mathscr{F} f\|_{p^{\prime}, \gamma} \leq \frac{M}{|c|}\left(\int_{0}^{2} d v(x)\right)^{1 / p}\|f\|_{2, v}^{2} .
$$


Now consider the particular function $f$ given by

$$
f(x)=\frac{|x|^{r}}{\sqrt{B(x)}} \mathbf{1}_{[-1,1]}(x)
$$

where $B$ is the function defined by $(1-1)$ and $\mathbf{1}_{[-1,1]}$ is the characteristic function of the interval $[-1,1]$. If $r>-(\alpha+1)$, this function belongs to $L^{1}(d v) \cap L^{2}(d v)$. From (1-4) we get

$$
\begin{aligned}
\mathscr{F} f(\lambda) & =\int_{0}^{1} x^{r+2 \alpha+1} j_{\alpha}(\lambda x) d x+\int_{0}^{1} x^{r+\alpha+1 / 2} \theta_{\lambda}(x) d x \\
& =\frac{1}{\lambda^{r+2 \alpha+2}} \int_{0}^{\lambda} x^{r+2 \alpha+1} j_{\alpha}(x) d x+\int_{0}^{1} x^{r+\alpha+1 / 2} \theta_{\lambda}(x) d x .
\end{aligned}
$$

Using the asymptotic expansion of the function $j_{\alpha}$ [Lebedev 1972; Watson 1944], given by

$$
j_{\alpha}(x)=\frac{2^{\alpha+1 / 2} \Gamma(\alpha+1)}{\sqrt{\pi} x^{\alpha+1 / 2}}\left(\cos \left(x-\alpha \frac{\pi}{2}-\frac{\pi}{4}\right)+O\left(\frac{1}{x}\right)\right) \text { as } x \rightarrow+\infty,
$$

we deduce that for $-(\alpha+1)<r<-\left(\alpha+\frac{1}{2}\right)$, the integral

$$
a:=\int_{0}^{+\infty} x^{r+2 \alpha+1} j_{\alpha}(x) d x
$$

exists and is finite, so

$$
\frac{1}{\lambda^{r+2 \alpha+2}} \int_{0}^{\lambda} x^{r+2 \alpha+1} j_{\alpha}(x) d x \sim \frac{a}{\lambda^{r+2 \alpha+2}} \quad \text { as } \lambda \rightarrow+\infty .
$$

On the other hand, for $\lambda>1$,

$$
\left|\int_{0}^{1} x^{r+\alpha+1 / 2} \theta_{\lambda}(x) d x\right| \leq \frac{c_{1}}{\lambda^{\alpha+3 / 2}} \int_{0}^{1} x^{r+\alpha+1 / 2} \Psi(x) d x,
$$

where

$$
\Psi(x)=\left(\int_{0}^{x}|Q(s)| d s\right) \exp \left(c_{2} \int_{0}^{x}|Q(s)| d s\right) \text { for all } x>0
$$

and $Q$ is given by $(1-5)$. Since $-(\alpha+1)<r<-\left(\alpha+\frac{1}{2}\right)$, we deduce that

$$
\mathscr{F} f(\lambda) \sim \frac{a}{\lambda^{r+2 \alpha+2}} \quad \text { as } \lambda \rightarrow+\infty .
$$

Using this and (1-6), it follows that there exist $K, R>0$ such that

$$
|\mathscr{F} f(\lambda)|^{p^{\prime}} \frac{1}{2 \pi|c(\lambda)|^{2}} \geq \frac{K}{\lambda p^{\prime}(r+2 \alpha+2)-2 \alpha-1} \quad \text { for } \lambda>R ;
$$


so for $r$ such that $p^{\prime}(r+2 \alpha+2)<2 \alpha+2$, we get

$$
\|\mathscr{F} f\|_{p^{\prime}, \gamma}^{p^{\prime}} \geq \int_{R}^{+\infty}|\mathscr{F} f(\lambda)|^{p^{\prime}} \frac{d \lambda}{2 \pi|c(\lambda)|^{2}} \geq \int_{R}^{+\infty} \frac{K}{\lambda p^{\prime}(r+2 \alpha+2)-2 \alpha-1} d \lambda=+\infty .
$$

This shows that the relation (6-3) is false if we choose $r$ so as to satisfy simultaneously the conditions $r>-(\alpha+1), r<-\left(\alpha+\frac{1}{2}\right)$ and

$$
r<-(2 \alpha+2)+\frac{2 \alpha+2}{p^{\prime}}
$$

This contradiction proves the lemma and Theorem 6.1.

\section{References}

[Chebli 1979] H. Chebli, "Théorème de Paley-Wiener associé à un opérateur différentiel singulier sur (0, $\infty)$ ", J. Math. Pures Appl. (9) 58:1 (1979), 1-19. MR 80g:47050 Zbl 0394.34019

[Folland 1984] G. B. Folland, Real analysis, Pure and Applied Mathematics, Wiley, New York, 1984. MR 86k:28001 Zbl 0549.28001

[Lebedev 1972] N. N. Lebedev, Special functions and their applications, Dover, New York, 1972. MR 50 \#2568 Zbl 0271.33001

[Nessibi et al. 1998] M. M. Nessibi, L. T. Rachdi, and K. Trimèche, "The local central limit theorem on the product of the Chébli-Trimèche hypergroup and the Euclidean hypergroup $\mathbb{R}^{n}$ ", J. Math. Sci. (Calcutta) 9:2 (1998), 109-123. MR 99m:60017

[Stein 1956] E. M. Stein, "Interpolation of linear operators", Trans. Amer. Math. Soc. 83 (1956), 482-492. MR 18,575d Zbl 0072.32402

[Stein and Weiss 1971] E. M. Stein and G. Weiss, Introduction to Fourier analysis on Euclidean spaces, Princeton mathematical series 32, Princeton University Press, Princeton, N.J., 1971. MR 46 \#4102 Zbl 0232.42007

[Trimèche 1981] K. Trimèche, "Transformation intégrale de Weyl et théorème de Paley-Wiener associés à un opérateur différentiel singulier sur $(0, \infty)$ ”, J. Math. Pures Appl. (9) 60:1 (1981), 51-98. MR 83i:47058 Zbl 0416.44002

[Trimèche 1997] K. Trimèche, "Inversion of the Lions transmutation operators using generalized wavelets”, Appl. Comput. Harmon. Anal. 4:1 (1997), 97-112. MR 98d:34046 Zbl 0872.34059

[Watson 1944] G. N. Watson, A treatise on the theory of Bessel functions, second ed., Cambridge University Press, Cambridge, 1944. MR 6,64a Zbl 0063.08184

[Weyl 1931] H. Weyl, The theory of groups and quantum mechanics, Methuen, London, 1931. Reprinted by Dover, New York, 1950. Zbl 0041.56804 JFM 58.1374.01

[Wong 1998] M. W. Wong, Weyl transforms, Universitext, Springer, New York, 1998. MR 2000c: 47098 Zbl 0908.44002

[Xu 1994] Z. Xu, Harmonic analysis on Chébli-Trimèche hypergroups, Ph.D. thesis, Murdoch University, Perth, Western Australia, 1994.

[Zeuner 1989] H. Zeuner, "The central limit theorem for Chébli-Trimèche hypergroups", J. Theoret. Probab. 2:1 (1989), 51-63. MR 90e:60087 Zbl 0912.60008

Received November 20, 2003. Revised April 7, 2004. 
CYRINE BACCAR

DEPARTMENT OF MATHEMATICS

FACULTY OF SCIENCES OF TUNIS

1060 TUNIS

TUNISIA

\section{LAKHDAR TANNECH RACHDI}

DEPARTMENT OF MATHEMATICS

FACULTY OF SCIENCES OF TUNIS 1060 TUNIS

TUNISIA

lakhdartannech.rachdi@fst.rnu.tn 